\title{
Fatores associados ao aleitamento cruzado
}

\author{
Factors associated with cross-nursing
}

Mariana Pujól von Seehausen ${ }^{1}$

Maria Inês Couto de Oliveira ${ }^{2}$

Cristiano Siqueira Boccolini ${ }^{3}$

${ }^{1}$ Programa de PósGraduação em Saúde

Coletiva, Instituto de Saúde Coletiva (ISC), Universidade Federal Fluminense (UFF) R. Marques de Paraná 303/ anexo $/ 4^{\circ}$ andar, Centro. 24033-900 Niterói RJ Brasil.

maripujol@hotmail.com

${ }^{2}$ Departamento de Epidemiologia e Bioestatística. ISC, UFF. Niterói RJ Brasil.

${ }^{3}$ Laboratório de Informação em Saúde, Instituto de Comunicação e Informação Científica e Tecnológica em Saúde, Fundação Oswaldo Cruz. Rio de Janeiro RJ Brasil.
Abstract This article aims to estimate the prevalence and analyze the factors associated with cross-nursing. A cross-sectional study was conducted in 2013 with interviews with a representative sample of mothers of infants less than one-year-old $(n '=695)$ attended in nine primary health units in Rio de Janeiro, Brazil. Sociodemographic characteristics were studied; pregnancy, childbirth and primary care assistance; maternal habits and baby features. Adjusted prevalence ratios (PR) were obtained by Poisson Regression, retaining variables associated with the outcome in the final model ( $p \leq 0.05)$. Cross-nursing was practiced by $29.4 \%$ of the mothers. Most practitioner mothers were relatives or friends. The following variables were directly associated with cross-nursing: being an adolescent mother ( $P R^{\prime}=$ 1.595), smoking $\left(P R^{\prime}=1.396\right)$, alcohol consumption $\left(P R^{\prime}=1.613\right)$, inappropriate baby feeding habits $(P R '=1.371)$ and infant's age in months $\left(P R^{\prime}=1.066\right)$. Maternal formal employment was inversely associated with the practice $\left(P R^{\prime}=\right.$ 0.579). Cross-nursing has a relevant prevalence among mothers assisted by primary health care units in Rio de Janeiro City. This issue should be addressed, especially among the most vulnerable groups, due to the association with adolescence and with unhealthy habits.

Key words Breast feeding, Cross-nursing, Primary Health Care, Cross-sectional studies, Child health
Resumo O objetivo deste artigo foi estimar a prevalência e analisar os fatores associados ao aleitamento cruzado. Estudo transversal conduzido em 2013 mediante entrevista com amostra representativa de mães de crianças $<1$ ano $(n=695) \mathrm{em}$ nove unidades básicas do Rio de Janeiro/RJ. Foram estudadas características sociodemográficas, da assistência à gravidez, ao parto e na atenção básica, hábitos maternos e características do bebê. Razões de prevalência ajustadas foram obtidas por regressão de Poisson, sendo mantidas no modelo final as variáveis que se associaram ao desfecho ( $p \leq 0,05)$. O aleitamento cruzado foi praticado por $29,4 \%$ das mães, geralmente entre parentes ou amigas. Mostraram-se diretamente associadas à prática: ser mãe adolescente $(R P=1,595)$, tabagismo $(R P=1,396)$, consumo de bebida alcoólica $(R P=1,613)$, regime inadequado de alimentação do bebê $(R P=1,371)$ e a idade do bebê em meses $(R P=1,066)$. O trabalho materno formal mostrou-se inversamente associado ao aleitamento cruzado $(R P=0,579)$. O aleitamento cruzado tem prevalência relevante entre mães assistidas por unidades primárias de saúde. Esse tema deve ser mais abordado, especialmente entre os grupos mais vulneráveis, devido à associação com a adolescência e com hábitos inadequados de saúde.

Palavras-chave Aleitamento materno, Aleitamento cruzado, Atenção Primária à Saúde, Estudos transversais, Saúde da criança 


\section{Introdução}

A amamentação possui diversas vantagens: estimula o crescimento e o desenvolvimento motor e cognitivo do bebê e tem impacto significativo na redução da morbi-mortalidade infantil ${ }^{1-3}$. Desde a década de 1980, quando foi lançado o Programa Nacional de Incentivo ao Aleitamento Materno, as políticas de estímulo, proteção e apoio ao aleitamento materno vêm resultando em aumento na duração mediana deste: de 2,5 meses em 1975 para 14,0 meses em $2006^{4-6}$.

$\mathrm{O}$ aleitamento cruzado consiste na prática em que uma mulher amamenta uma criança que não é seu filho, um compartilhamento informal entre mães, não remunerado, podendo ser recíproco. É considerado ocasional, pois a mãe continua amamentando seu próprio filho e geralmente ocorre em situações em que a criança está sob o cuidado de uma outra lactante ${ }^{7-9}$. Esta prática é culturalmente aceita no Brasil, possivelmente devido à tradição das amas de leite, que no século XIX amamentavam e cuidavam das crianças de famílias com as quais viviam ${ }^{10}$.

Segundo a Organização Mundial da Saúde (OMS), nas excepcionais condições em que o leite de uma mulher não é adequado ou não está disponível para a alimentação de seu filho, a escolha da melhor alternativa depende de circunstâncias individuais ou coletivas, como catástrofes. Tais alternativas são: amamentação realizada por uma lactante saudável, leite ordenhado de outra lactante, leite obtido em bancos de leite humano ou substituto ao leite humano ${ }^{11,12}$. No Brasil, o aleitamento cruzado é formalmente contraindicado pelo Ministério da Saúde, desde 1996, devido ao risco de transmissão vertical do HIV pela amamentação ${ }^{13}$.

O aleitamento cruzado é um tema pouco estudado pela literatura. Embora desaconselhado no Brasil há cerca de 20 anos, não foram encontrados artigos que tenham descrito a prevalência desta prática e investigado os seus determinantes. Não se sabe, portanto, se a prática do aleitamento cruzado tem se mantido, se foi reduzida, ou mesmo se expandiu no período. Este trabalho teve por objetivo estimar a prevalência de aleitamento cruzado em mulheres assistidas por unidades básicas de saúde e analisar os fatores associados à prática.

\section{Métodos}

Estudo transversal, cuja população foi composta por uma amostra representativa das mães de crianças menores de um ano assistidas pelas nove unidades básicas do Sistema Único de Saúde (SUS) que possuíam Postos de Recebimento de Leite Humano Ordenhado (PRLHO) no município do Rio de Janeiro em 2013. Dentre os nove PRLHO, seis funcionavam em Clínicas da Família e três em Centros Municipais de Saúde, distribuídos em cinco das dez áreas de planejamento da cidade $^{14}$. O presente estudo faz parte da pesquisa "Avaliação dos fatores associados à doação de leite materno por usuárias de unidades básicas de saúde da cidade do Rio de Janeiro" e contou com apoio da FAPERJ. Foram selecionadas para o estudo unidades básicas com PRLHO, pois nestas unidades as mães teriam a possibilidade de doar seu leite de uma forma institucional.

O projeto de pesquisa foi desenhado para a investigação de vários desfechos relativos à doação de leite humano e à amamentação e o tamanho da amostra original foi definido com base em uma prevalência de $50 \%$ dos desfechos (pois garante o maior tamanho de amostra possível para nível de erro e confiança controlados), com alfa de $5 \%$ e beta de $80 \%$ para detectar uma razão de chances de 1,5, resultando em uma amostra de 697 sujeitos $^{15}$. A quantidade de mães entrevistadas em cada unidade foi proporcional à média do número de crianças menores de um ano acompanhadas pela unidade no bimestre anterior ao trabalho de campo.

As fontes de dados foram questionários estruturados aplicados às mães nos meses de novembro e dezembro de 2013. Os instrumentos foram testados previamente em estudo piloto, conduzido nos meses de setembro e outubro de 2013, em duas unidades básicas do município do Rio de Janeiro. Entrevistadoras, nutricionistas e enfermeiras, foram capacitadas para a aplicação dos questionários de forma padronizada em treinamento teórico-prático com carga horária de 20 horas e participaram do estudo piloto. O trabalho de campo contou com a supervisão de uma profissional com prática em pesquisa e dos pesquisadores envolvidos. Em cada unidade básica de saúde a entrevistadora correspondente coletou dados em todos os turnos de atendimen- 
to até que o tamanho amostral correspondente fosse atingido. O período de coleta de dados em cada unidade variou de 25 dias a um mês e meio.

Foram consideradas como variáveis de exposição as características sociodemográficas maternas e contextuais (idade, cor da pele, escolaridade, renda materna, paridade, experiência prévia de amamentar, local de moradia, presença de companheiro, chefe da família, morar com sogra ou mãe e número de moradores na residência), características da assistência recebida durante a gravidez e o parto e do recém-nascido (número de consultas pré-natais, local de realização do pré-natal, orientação sobre aleitamento materno durante o pré-natal, tipo de hospital, tipo de parto, idade gestacional do bebê, peso ao nascer, internação em unidade neonatal, primeira mamada na primeira hora de vida e tipo de aleitamento na alta hospitalar) e os hábitos e atividades maternas, a assistência na unidade básica e características do bebê (fumar pelo menos um cigarro por dia, ingerir bebida alcoólica pelo menos uma vez por semana, percepção da própria saúde, estar estudando, exercer trabalho remunerado formal ou informal, percepção de ajuda para amamentar, sexo do bebê, idade do bebê em meses, número de consultas do bebê, cuidador principal, estar na creche, regime adequado de alimentação do bebê, uso de mamadeira e uso de chupeta). A variável "regime adequado de alimentação do bebê" foi construída a partir de informações sobre a idade deste e o tipo de alimentação recebida. O regime alimentar foi considerado adequado quando o bebê menor de seis meses de idade estava em aleitamento materno exclusivo (AME) ou quando a criança no segundo semestre de vida recebia leite materno complementado por fruta e/ou suco de frutas e por papa salgada e/ou comida de panela.

Os dados sobre o aleitamento cruzado foram obtidos por meio de dois conjuntos de perguntas: 1. "Desde que o/a (nome do bebê) nasceu, você amamentou alguma outra criança sem ser seu filho/a?"; "Esta(s) crianças era(m) filha(s) de quem?"; "Por que você amamentou estas criança(s)?” e 2. "O/a (nome do bebê) foi amamentado alguma vez por outra pessoa?”; “Quem era(m) esta(s) mulheres que amamentou(aram) o/a (nome do bebê)?; "Por que esta mulher amamentou (nome do bebê)?”. No entanto, como o perfil das mães que praticaram as duas formas de aleitamento cruzado (a mãe ter amamentado outra criança que não o seu filho e a mãe ter o seu filho amamentado por outra nutriz) foi semelhante (dados não expostos), optou-se por empregar um desfecho único: o aleitamento cruzado, que compreendeu as duas formas referidas.

Análise univariada foi desenvolvida para conhecimento da distribuição das variáveis de exposição e do desfecho sob investigação, a prática do aleitamento cruzado. Em seguida foi conduzida uma análise bivariada entre cada variável de exposição e o desfecho, utilizando-se teste quiquadrado de Pearson, com intervalos de confiança de 95\%. As variáveis que obtiveram valor de $\mathrm{p}$ $\leq 0,20$ foram incluídas na modelagem estatística.

As razões de prevalência ajustadas foram obtidas por modelo de regressão de Poisson com variância robusta, pois o desfecho apresentou prevalência elevada ${ }^{16}$. O modelo final, utilizado para estimar medidas de associação com seus respectivos intervalos de confiança de 95\%, foi composto pelas variáveis de exposição que obtiveram valor de $\mathrm{p} \leq 0,05$. A regressão seguiu modelo conceitual hierarquizado, segundo a proximidade temporal de cada variável com o desfecho $^{17}$. Foram consideradas como variáveis distais as características maternas e contextuais, como variáveis intermediárias as características do prénatal, do parto, do recém-nascido e da assistência hospitalar e como variáveis proximais os hábitos e as atividades maternas, a assistência na unidade básica e as características do bebê.

\section{Resultados}

A prevalência de aleitamento cruzado entre as mães foi de 29,4\% ( $n=204)$, sendo que 10,8\% (n = 75) amamentaram uma ou mais crianças que não o seu filho, 9,8\% $(n=68)$ tiveram seu filho amamentado por uma ou mais nutrizes e $8,8 \%$ $(\mathrm{n}=61)$ praticaram as duas formas de aleitamento cruzado.

Mais da metade das mães praticantes $(56,4 \%)$ tinha relação de parentesco com a outra nutriz envolvida e a principal motivação para a prática foi o bebê estar sendo cuidado por uma que não sua mãe $(51,5 \%)$ (Tabela 1$)$.

Entre as mães entrevistadas, 22,7\% eram adolescentes, $73,5 \%$ se autodeclararam de cor não branca e 77,6\% tinham ensino fundamental incompleto (Tabela 2). Fizeram o pré-natal em unidade básica de saúde 79,1\% das mães, 78,7\% foram orientadas sobre aleitamento materno, $41,7 \%$ dos partos foram operatórios e $16,3 \%$ dos bebês ficaram internados em unidade neonatal (Tabela 3). Em relação aos hábitos maternos, $11,1 \%$ das mães fumavam (pelo menos um cigarro por dia), 11,7\% consumiam bebida alcoólica 


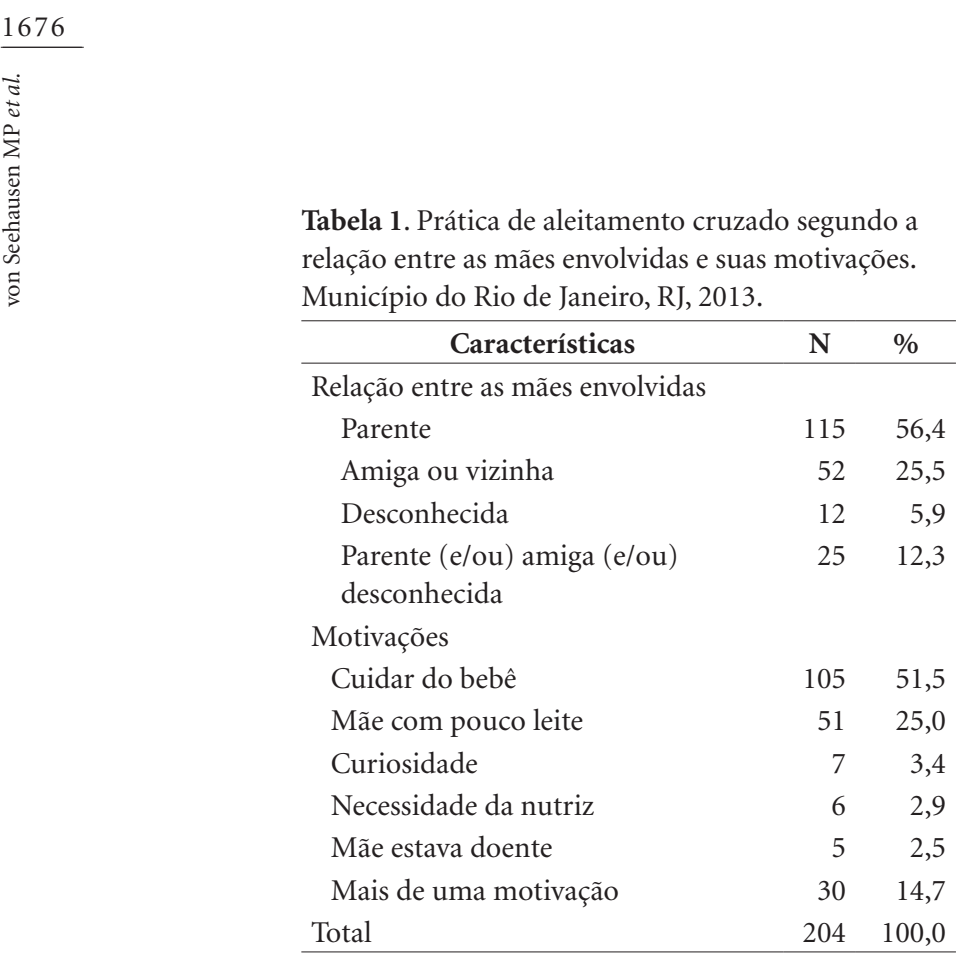

(em geral, ocasionalmente) e 17,4\% percebia sua saúde como ruim ou regular. Foram orientadas sobre a ordenha manual das mamas $18,7 \%$ das mães. A grande maioria das mães $(89,5 \%)$ era a principal cuidadora de seu filho, $8,9 \%$ delas estudavam e 63,2\% não trabalhavam (Tabela 4).

$\mathrm{Na}$ análise bivariada, mostraram-se associadas ao aleitamento cruzado as variáveis distais, idade, cor da pele, escolaridade e renda maternas, local de moradia e viver com companheiro ( Tabela 2), as intermediárias, local de realização do pré-natal e orientação sobre aleitamento materno durante o mesmo (Tabela 3), e as proximais, hábito de fumar, consumo de bebida alcoólica, percepção da própria saúde, trabalho materno atual, percepção de ajuda para amamentar, alimentação adequada, uso de mamadeira e idade do bebê à época da entrevista (Tabela 4).

No modelo múltiplo hierarquizado, mostraram-se diretamente associados ao aleitamento cruzado: ser mãe adolescente $(\mathrm{RP}=1,595)$, hábito de fumar $(\mathrm{RP}=1,396)$, consumo de bebida alcoólica $(\mathrm{RP}=1,613)$, regime inadequado de alimentação do bebê $(\mathrm{RP}=1,371)$ e a idade deste em meses $(R P=1,066)$. O trabalho materno formal mostrou-se inversamente associado à prática $(\mathrm{RP}=0,579)($ Tabela 5).

\section{Discussão}

Embora desaconselhado no Brasil pelo Ministério da Saúde há duas décadas, quase um terço das mães entrevistadas praticou o aleitamento cruza- $\mathrm{do}^{13}$. Esta prevalência foi próxima à encontrada em estudo transversal conduzido em 2008 com 120 mães que tiveram seus filhos em maternidade pública do município de Horizonte/Ceará $(32,0 \%)$ e inferior à encontrada em usuárias do SUS de duas cidades de médio porte do Rio de Janeiro em estudo de coorte conduzido de 2008 a $2010(39,5 \%)^{18,19}$.

Observou-se que, na maioria das vezes, as mães praticantes eram parentes ou amigas e o aleitamento cruzado ocorria por ocasião do cuidado do bebê por uma nutriz que não a sua mãe. Estudos em outros cenários também observaram tal comportamento. Entre as mais de 70\% das mães que praticaram o aleitamento cruzado em Horizonte/Ceará, este ocorreu entre parentes ou amigas e em ocasiões em que a mãe estava distante e o bebê sob os cuidados de outra nutriz ${ }^{18}$. Thorley $^{8}$ verificou que, dentre 43 australianas praticantes do aleitamento cruzado captadas pela internet, $45,3 \%$ realizavam a prática com irmãs ou cunhadas e $34,0 \%$ com amigas. No trabalho de Krantz e Kupper ${ }^{7}$ três mães americanas praticantes do aleitamento cruzado entre si relataram que a prática geralmente ocorria quando uma delas estava cuidando do bebê de uma das amigas, aliviando a preocupação com a alimentação da criança no caso da ausência materna.

No Brasil, vem sendo desenvolvida uma política de promoção ao aleitamento materno há mais de 30 anos, que vem sensibilizando as mães sobre a importância da amamentação e do leite materno para a saúde de seus filhos ${ }^{4}$. Como nem sempre as mães conseguem estar próximas de seus filhos ou adquirir as habilidades necessárias à ordenha do leite materno para a sua administração por outra pessoa, é possível que o aleitamento cruzado, para elas, pareça ser uma forma viável de manter a amamentação e o fornecimento de leite humano ao bebê, possivelmente pelo seu desconhecimento do risco de transmissão vertical de HIV e outras doenças pelo leite hu$\operatorname{mano}^{20}$.

$\mathrm{Na}$ análise múltipla, a prevalência de aleitamento cruzado aumentou 6,6\% a cada mês de vida do bebê. Também se mostraram diretamente associados ao aleitamento cruzado: adolescência, hábito de fumar, consumo de bebida alcoólica, não dispor de trabalho materno formal, e oferecer a seus filhos um regime alimentar inadequado. Logo, o aleitamento cruzado parece estar relacionado a uma maior vulnerabilidade materna, pela idade das mães que o praticam, pela ausência de direitos trabalhistas e por estas não seguirem as orientações preconizadas pelo 
Tabela 2. Prevalência e razão de prevalência bruta de aleitamento cruzado segundo características maternas e contextuais. Município do Rio de Janeiro, 2013.

\begin{tabular}{|c|c|c|c|c|c|}
\hline Características & $\mathbf{N}$ & $\%$ & $\% \mathrm{AC}$ & RP & p-valor \\
\hline \multicolumn{6}{|l|}{ Idade da mãe } \\
\hline 20 anos ou mais & 537 & 77,3 & 25,5 & 1 & \\
\hline 13 a 19 anos & 158 & 22,7 & 42,4 & 1,662 & $<0,001$ \\
\hline \multicolumn{6}{|l|}{ Cor da pele } \\
\hline Branca & 184 & 26,5 & 25,0 & 1 & \\
\hline Não branca & 510 & 73,5 & 31,0 & 1,239 & 0,127 \\
\hline \multicolumn{6}{|l|}{ Escolaridade materna } \\
\hline 8 anos completos ou mais & 539 & 77,6 & 27,8 & 1 & \\
\hline Até 7 anos completos & 156 & 22,4 & 34,6 & 1,244 & 0,101 \\
\hline \multicolumn{6}{|l|}{ Renda materna } \\
\hline 1 salário mínimo ou mais & 202 & 29,3 & 20,3 & 1 & \\
\hline Menos de 1 salário mínimo ou sem renda & 487 & 70,7 & 33,3 & 1,639 & 0,001 \\
\hline \multicolumn{6}{|l|}{ Paridade } \\
\hline Primípara & 328 & 47,2 & 30,8 & 1 & \\
\hline Multípara & 367 & 52,8 & 28,1 & 0,911 & 0,431 \\
\hline \multicolumn{6}{|l|}{ Experiência com AM } \\
\hline Nunca amamentou antes & 347 & 49,9 & 30,3 & 1 & \\
\hline Amamentou menos de 1 ano & 176 & 25,3 & 29,5 & 0,976 & 0,866 \\
\hline Amamentou mais de 1 ano & 172 & 24,7 & 27,3 & 0,903 & 0,489 \\
\hline \multicolumn{6}{|l|}{ Local de moradia } \\
\hline Bairro & 252 & 36,3 & 23,0 & 1 & \\
\hline Comunidade & 443 & 63,7 & 33,0 & 1,432 & 0,006 \\
\hline \multicolumn{6}{|l|}{ Vive com companheiro } \\
\hline Sim & 547 & 78,7 & 28,2 & 1 & \\
\hline Não & 148 & 21,3 & 33,8 & 1,200 & 0,182 \\
\hline \multicolumn{6}{|l|}{ Chefe da família } \\
\hline Outros & 594 & 85,5 & 28,5 & 1 & \\
\hline Própria mulher & 101 & 14,5 & 34,7 & 1,218 & 0,206 \\
\hline \multicolumn{6}{|l|}{ Mora com mãe ou sogra } \\
\hline Não & 538 & 77,4 & 28,4 & 1 & \\
\hline Mãe ou sogra ou ambas & 157 & 22,6 & 32,5 & 1,142 & 0,327 \\
\hline \multicolumn{6}{|l|}{ Número de moradores } \\
\hline Até 4 moradores & 437 & 62,9 & 29,1 & 1 & \\
\hline De 5 a 11 moradores & 258 & 37,1 & 29,8 & 1,027 & 0,827 \\
\hline Total & 695 & 100,0 & 29,4 & - & - \\
\hline
\end{tabular}

Ministério da Saúde quanto a hábitos de vida e de alimentação do bebê.

Adolescentes costumam ter relações de amizade bastante estáveis, passando boa parte do seu tempo em atividades de socialização com os amigos, compartilhando sentimentos e experiências, sendo a confiança uma característica bastante valorizada ${ }^{21}$. Mulheres jovens costumam procurar redes de apoio informal para lidar com os desafios da gravidez e da maternidade, tanto proporcionando apoio quanto o recebendo ${ }^{22}$. Estas características da mãe adolescente podem 


\begin{tabular}{|c|c|c|c|c|c|c|}
\hline \multirow[t]{33}{*}{ 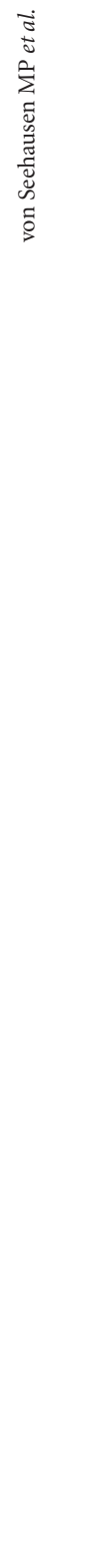 } & $\begin{array}{l}\text { Tabela 3. Prevalência e razão de } \\
\text { do parto, do recém-nato e da as }\end{array}$ & $\begin{array}{l}\text { de al } \\
\text { r. } \mathrm{Mu}\end{array}$ & $\begin{array}{l}\text { ento } \mathrm{cr} \\
\text { io do } \mathrm{Ri}\end{array}$ & $\begin{array}{l}\text { segundo } \\
\text { aneiro, } 2\end{array}$ & cterística & pré-natal, \\
\hline & Características & $\mathbf{N}$ & $\%$ & $\% \mathrm{AC}$ & RP & p-valor \\
\hline & \multicolumn{6}{|l|}{ Consultas de pré-natal } \\
\hline & 6 ou mais consultas & 614 & 88,5 & 30,1 & 1 & \\
\hline & 0 a 5 consultas & 80 & 11,5 & 23,8 & 0,788 & 0,239 \\
\hline & \multicolumn{6}{|l|}{ Local de realização do pré-natal } \\
\hline & Unidade básica do SUS & 545 & 79,1 & 31,2 & 1 & \\
\hline & Outro serviço & 144 & 20,9 & 22,9 & 0,735 & 0,053 \\
\hline & \multicolumn{6}{|c|}{ Orientação sobre AM no pré-natal } \\
\hline & Sim & 547 & 78,7 & 27,8 & 1 & \\
\hline & Não & 148 & 21,3 & 35,1 & 1,264 & 0,082 \\
\hline & \multicolumn{6}{|l|}{ Hospital de nascimento } \\
\hline & Não credenciado & 490 & 71,0 & 30,2 & 1 & \\
\hline & Hospital Amigo Criança & 200 & 29,0 & 27,0 & 0,894 & 0,401 \\
\hline & \multicolumn{6}{|l|}{ Tipo de parto } \\
\hline & Normal & 405 & 58,3 & 30,6 & 1 & \\
\hline & Cesariana & 290 & 41,7 & 27,6 & 0,901 & 0,387 \\
\hline & \multicolumn{6}{|l|}{ Idade gestacional } \\
\hline & A termo & 624 & 89,8 & 29,5 & 1 & \\
\hline & Pré-termo & 71 & 10,2 & 28,2 & 0,955 & 0,817 \\
\hline & \multicolumn{6}{|l|}{ Peso ao nascer } \\
\hline & $2500 \mathrm{~g}$ ou mais & 652 & 93,8 & 29,1 & 1 & \\
\hline & $<2500 \mathrm{~g}$ & 43 & 6,2 & 32,6 & 1,117 & 0,634 \\
\hline & \multicolumn{6}{|l|}{ Internação do bebê } \\
\hline & Não & 582 & 83,7 & 29,4 & 1 & \\
\hline & Sim & 113 & 16,3 & 29,2 & 0,994 & 0,970 \\
\hline & \multicolumn{6}{|l|}{ Primeira mamada } \\
\hline & Após a primeira hora & 300 & 43,2 & 27,0 & 1 & \\
\hline & $\mathrm{Na}$ primeira hora & 395 & 56,8 & 31,1 & 1,153 & 0,235 \\
\hline & \multicolumn{6}{|l|}{ Tipo de AM na alta } \\
\hline & AME & 549 & 79,0 & 28,6 & 1 & \\
\hline & Não AME & 146 & 21,0 & 32,2 & 1,126 & 0,397 \\
\hline & Total & 695 & 100,0 & 29,4 & - & - \\
\hline
\end{tabular}

${ }^{*} \mathrm{RP}=$ razão de prevalência bruta; $\mathrm{AC}=$ aleitamento cruzado; $\mathrm{AM}=$ aleitamento materno.

ras, estando associado a celebrações, situações de negócio e sociais, cerimônias religiosas e eventos culturais $^{24}$. Embora tal consumo seja contraindicado durante a gestação e a lactação, Oliveira e Simões $^{25}$ observaram que $10 \%$ das gestantes frequentadoras de unidade de saúde no Estado do Rio de Janeiro ingeriam bebidas alcoólicas moderadamente, especialmente em festas. Tanto as mães fumantes quanto as que ingeriam bebidas alcoólicas apresentaram uma maior prevalência (40\% e 60\%, respectivamente) de aleitamento cruzado, apontando, da mesma forma, para a dimensão social e de suporte mútuo da prática.

Em resultados preliminares de estudo de coorte realizado em dois municípios do Rio de
Janeiro, o trabalho informal aumentou a prevalência do aleitamento cruzado em $25 \%^{19}$. No presente estudo, esta categoria de variável sinalizou o mesmo tipo de efeito, porém não alcançou significância estatística. Já o trabalho materno formal reduziu a prática do aleitamento cruzado em $42 \%$, provavelmente porque estas mulheres dispõem de licença maternidade e de outros direitos trabalhistas que protegem o aleitamento materno.

Em estudo realizado em Porto Alegre, práticas recomendadas de aleitamento materno e de alimentação complementar saudável foram mais frequentes entre mães que seguiam as recomendações dos profissionais de saúde e que enten- 
Tabela 4. Prevalência e razão de prevalência bruta de aleitamento cruzado segundo hábitos e atividades maternas, assistência na unidade básica e características do bebê. Município do Rio de Janeiro, 2013.

\begin{tabular}{|c|c|c|c|c|c|}
\hline Características & $\mathbf{N}$ & $\%$ & $\% \mathrm{AC}$ & $\mathbf{R P}$ & p-valor \\
\hline \multicolumn{6}{|l|}{ Fuma } \\
\hline Não & 617 & 88,9 & 27,2 & 1 & \\
\hline Sim & 77 & 11,1 & 46,8 & 1,717 & $<0,001$ \\
\hline \multicolumn{6}{|l|}{ Bebida alcoólica } \\
\hline Não & 614 & 88,3 & 26,7 & 1 & \\
\hline Sim & 81 & 11,7 & 49,4 & 1,849 & $<0,001$ \\
\hline \multicolumn{6}{|l|}{ Percepção da sua saúde } \\
\hline Boa & 574 & 82,6 & 26,8 & 1 & \\
\hline Ruim ou regular & 121 & 17,4 & 41,3 & 1,540 & 0,001 \\
\hline \multicolumn{6}{|l|}{ Estudante } \\
\hline Não & 632 & 91,1 & 30,1 & 1 & \\
\hline $\operatorname{Sim}$ & 62 & 8,9 & 22,6 & 0,751 & 0,217 \\
\hline \multicolumn{6}{|l|}{ Trabalho materno atual } \\
\hline Não trabalha & 439 & 63,2 & 32,3 & 1 & \\
\hline Trabalho formal & 177 & 25,5 & 15,8 & 0,466 & $<0,001$ \\
\hline Trabalho informal & 79 & 11,4 & 43,0 & 1,559 & 0,005 \\
\hline \multicolumn{6}{|l|}{ Percepção de ajuda para amamentar } \\
\hline $\operatorname{Sim}$ & 360 & 51,8 & 26,1 & 1 & \\
\hline Não ou regular & 335 & 48,2 & 32,8 & 1,258 & 0,052 \\
\hline \multicolumn{6}{|l|}{ Orientação sobre ordenha manual } \\
\hline Sim & 130 & 18,7 & 25,4 & 1 & \\
\hline Não & 565 & 81,3 & 30,3 & 1,192 & 0,271 \\
\hline \multicolumn{6}{|l|}{ Cuidador } \\
\hline Outra pessoa & 73 & 10,5 & 27,4 & 1 & \\
\hline Mãe & 621 & 89,5 & 29,6 & 1,081 & 0,692 \\
\hline \multicolumn{6}{|l|}{ Bebê na creche } \\
\hline Não & 683 & 98,3 & 29,6 & 1 & \\
\hline Sim & 12 & 1,7 & 16,7 & 0,564 & 0,330 \\
\hline \multicolumn{6}{|l|}{ Alimentação adequada } \\
\hline Não & 350 & 50,4 & 35,4 & 1 & \\
\hline Sim & 345 & 49,6 & 23,2 & 0,655 & $<0,001$ \\
\hline \multicolumn{6}{|l|}{ Uso de mamadeira } \\
\hline Não & 295 & 42,4 & 23,1 & 1 & \\
\hline Sim & 400 & 57,6 & 34,0 & 1,475 & 0,002 \\
\hline \multicolumn{6}{|l|}{ Uso de chupeta } \\
\hline Não & 384 & 55,3 & 28,6 & 1 & \\
\hline Sim & 310 & 44,7 & 30,3 & 1,059 & 0,630 \\
\hline \multicolumn{6}{|l|}{ Sexo do bebê } \\
\hline Feminino & 323 & 53,5 & 27,9 & 1 & \\
\hline Masculino & 372 & 46,5 & 30,6 & 1,100 & 0,422 \\
\hline \multicolumn{6}{|l|}{ Idade do bebê } \\
\hline 0 a 5 meses & 429 & 61,7 & 23,8 & 1 & \\
\hline 6 até 12 meses & 266 & 38,3 & 38,3 & 1,613 & $<0,001$ \\
\hline Total & 695 & 100,0 & 29,4 & - & - \\
\hline
\end{tabular}

${ }^{*} \mathrm{RP}=$ razão de prevalência bruta; $\mathrm{AC}=$ aleitamento cruzado

diam a importância da alimentação adequada para seus filhos, porém cerca de $50 \%$ das mães não seguiam estas recomendações ${ }^{26}$. Embora 


\begin{tabular}{|c|c|c|c|c|}
\hline \multirow[t]{20}{*}{ 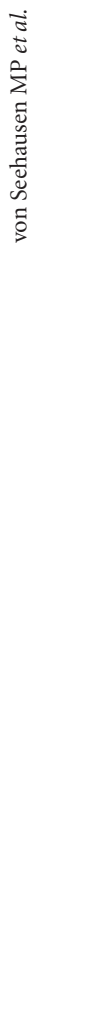 } & $\begin{array}{l}\text { Tabela 5. Fatores associados ao a } \\
\text { Município do Rio de Janeiro, } 20\end{array}$ & mães a & unidades & le saúde. \\
\hline & Características & $\mathbf{R P}_{\mathbf{a}}$ & p-valor & IC95\% \\
\hline & \multicolumn{4}{|l|}{ Idade da mãe } \\
\hline & 20 anos ou mais & 1 & & \\
\hline & 13 a 19 anos & 1,595 & $<0,001$ & $1,276-1,993$ \\
\hline & \multicolumn{4}{|l|}{ Hábito de fumar } \\
\hline & Não & 1 & & \\
\hline & Sim & 1,396 & 0,021 & $1,051-1,854$ \\
\hline & \multicolumn{4}{|l|}{ Consumo de bebida alcoólica } \\
\hline & Não & 1 & & \\
\hline & Sim & 1,613 & 0,001 & $1,228-2,119$ \\
\hline & \multicolumn{4}{|l|}{ Trabalho materno atual } \\
\hline & Não trabalha & 1 & & \\
\hline & Trabalho formal & 0,579 & 0,004 & $0,400-0,838$ \\
\hline & Trabalho informal & 1,272 & 0,082 & $0,970-1,668$ \\
\hline & \multicolumn{4}{|l|}{ Alimentação adequada } \\
\hline & Sim & 1 & & \\
\hline & Não & 1,371 & 0,007 & $1,089-1,726$ \\
\hline & \multicolumn{4}{|l|}{ Idade do bebê } \\
\hline & Idade do bebê em meses & 1,066 & $<0,001$ & $1,032-1,100$ \\
\hline
\end{tabular}

${ }^{\star} \mathrm{RP}_{\mathrm{a}}=$ razão de prevalência ajustada.

difíceis de entender ou conflitantes. As atitudes maternas em relação ao tipo de alimentação a ser oferecida aos seus filhos são fortemente influenciadas por familiares, amigos e parceiros ${ }^{27,28}$. No presente estudo, o aleitamento cruzado foi mais praticado por mães que não seguiam as recomendações preconizadas quanto à alimentação de seus filhos, possivelmente por não receberem e/ou não compreenderem as informações fornecidas pelos profissionais de saúde ou por darem preferência a conselhos fornecidos por familiares e amigos.

Foi interessante observar que nenhuma variável relativa à assistência pré-natal, ao parto ou à díade mãe-bebê se mostrou associada ao aleitamento cruzado. O presente estudo não investigou se as mães entrevistadas foram orientadas pela equipe de saúde sobre a contraindicação à prática do aleitamento cruzado. No entanto, estas orientações parecem ser pouco frequentes e pontuais. Vieira et al. ${ }^{29}$, em estudo qualitativo com 17 puérperas internadas em hospital filantrópico de Ribeirão Preto/SP, encontraram que nenhuma delas havia sido orientada sobre o tema no prénatal.

Não foi encontrada associação entre o aleitamento cruzado e as características do bebê, como o nascimento pré-termo e o baixo peso ao nascer, indicando que não foi a vulnerabilidade da criança que se associou a esta prática, e sim as características maternas. As atitudes de uma mulher frente à amamentação estruturam-se a partir da interação com o recém-nascido, a comunidade, seus familiares e suas atividades diárias, sendo a amamentação socioculturalmente condicionada, impregnada por ideologias e determinantes que resultam das condições concretas da vida ${ }^{30,31}$. A mera transmissão de informação dos profissionais de saúde para as mães não costuma ser suficiente para determinar as ações destas em relação às práticas alimentares de seu filho ${ }^{26}$. Portanto, é possível assumir que cartazes e instruções esporádicas contraindicando o aleitamento cruzado podem não ser suficientes para impedi-lo, enquanto prática social impregnada pela cultura.

É importante ressaltar que o presente estudo apresenta limitações. Pelo seu delineamento transversal, não se dispõe de informação sobre o momento de início e de término da prática do aleitamento cruzado, impossibilitando o estabelecimento de relação causal entre o desfecho e a maior parte das variáveis proximais investigadas. Além disso, a única fonte de informação disponível sobre a prática foi a entrevista materna, o que inviabiliza a estimação de possível viés de informação, uma vez que não existem registros oficiais 
sobre o aleitamento cruzado e o mesmo não foi observado diretamente. A escassez de dados sobre a prática na literatura também limita a possibilidade de comparação com outros contextos, uma vez que mais estudos sobre o aleitamento cruzado enriqueceriam a discussão do tema.

Conclui-se que, embora contraindicada pelo Ministério da Saúde e negligenciada pela literatura, a prática do aleitamento cruzado tem prevalência relevante entre mães assistidas pelo SUS no contexto da cidade do Rio de Janeiro e ocorre especialmente entre mães próximas e na ocasião do cuidado do bebê por outra nutriz. Tal prevalência aumenta com a idade do bebê e é maior entre mães mais vulneráveis: jovens, adeptas de hábitos não saudáveis e não trabalhadoras formais.

É importante que futuros estudos investiguem o conhecimento materno sobre aleitamen- to cruzado e a cobertura e o teor das orientações prestadas sobre o tema na assistência pré-natal, ao parto e à díade mãe-filho em diferentes contextos e cenários. Sugere-se, também, que sejam conduzidos estudos longitudinais que aprofundem a investigação de fatores associados ao aleitamento cruzado e que explorem aspectos não abordados no presente trabalho, como a duração da prática do aleitamento cruzado e a sua extensão para além das frequentadoras do Sistema Único de Saúde.

Recomenda-se, portanto, que a prática do aleitamento cruzado seja mais estudada e se torne objeto de reflexão pelos profissionais das instituições de saúde e pela sociedade como um todo, seja para desestimulá-la de forma efetiva, por meio de aconselhamento, seja para reavaliá -la e aceitá-la em determinadas circunstâncias e contextos, como sugerido pela OMS.

\section{Colaboradores}

MP von Seehausen e MIC Oliveira foram responsáveis pela concepção geral do estudo, pela análise e interpretação dos dados, pela redação do artigo e pela aprovação final da versão a ser publicada. CS Boccolini participou da análise e interpretação dos dados e da redação final da versão a ser publicada. 


\section{Referências}

1. Dewey KG, Cohen RJ, Brown KH, Rivera LL. Effects of exclusive breastfeeding for four versus six months on maternal nutritional status and infant motor development: results of two randomized trials in Honduras. $J$ Nutr 2001; 131(2):262-267.

2. Mortensen EL, Michaelsen KF, Sanders SA, Reinisch JM. The association between duration of breastfeeding and adult intelligence. JAMA 2002; 287(18):2365-2371.

3. Boccolini CS, Boccolini PM, Carvalho ML, Oliveira MI. Exclusive breastfeeding and diarrhea hospitalization patterns between 1999 and 2008 in Brazilian State Capitals. Cien Saude Colet 2012; 17(7):1857-1863.

4. Rea MF. A review of breastfeeding in Brazil and how the country has reached ten months' breastfeeding duration. Cad Saude Publica. 2003; 19(Supl. 1):S37-45.

5. Venancio SI, Monteiro CA. Breast-feeding trends between 1970 and 1980 in Brazil. Rev Bras Epidemiol 1998; 1(1):40-49.

6. Brasil. Ministério da Saúde (MS). Pesquisa Nacional de Demografia e Saúde da Criança e da Mulher (PNDS) 2006: dimensões do processo reprodutivo e da saúde da criança. Brasília: MS; 2009.

7. Krantz JZ, Kupper NS. Cross-nursing: wet nursing in a contemporary context. Pediatrics 1981; 67(5):715-717.

8. Thorley V. Mothers' experiences of sharing breastfeeding or breastmilk: co-feeding in Australia 1978-2008. Breastfeed Rev 2009; 17(1):9-18.

9. La Leche League Internacional. Wet nursing and Cross nursing [internet] [cited 2015 Jul 10]. Available from: http://www.lalecheleague.org/llleaderweb/lv/lvjulaug95p53.html

10. Koutsoukos SSM. 'Amas mercenárias': o discurso dos doutores em medicina e os retratos de amas - Brasil, segunda metade do século XIX. Hist. Cienc. Saude-Manguinhos 2009; 16(2):305-324.

11. World Health Organization (WHO), UNICEF, Pan American Health Organization. Haiti earthquake: Technical note on HIV and infant feeding [Internet]. Genebra: WHO; 2010. [cited 2015 Jun 10]. Available from: http://www.unicef.org/lac/comun._conjunto.pdf

12. World Health Organization (WHO). Global strategy for infant and young child feeding. Genebra: WHO; 2003. [cited 2015 Jun 10]. Available from: http://www. who.int/nutrition/publications/gs_infant_feeding_ text_eng.pdf

13. Brasil. Ministério da Saúde (MS). Portaria n 2.415, de 12 de dezembro de 1996. Dispõe sobre medidas para prevenção da contaminação pelo HIV pelo aleitamento materno. Diário Oficial da União 1996; 13 dez.

14. Rio de Janeiro. Secretaria Municipal de Saúde (SMS). Gerência de Programas de Saúde da Criança. Projeto Piloto de Implantação dos Entrepostos de Coleta de Leite Humano na Rede Básica de Saúde. Rio de Janeiro: SMS; 2009.

15. Cochran WG. Sampling Techniques. New York: John Wiley \& Sons; 1977.

16. Coutinho LMS, Scazufca M, Menezes PR. Methods for estimating prevalence ratios in crosssectional studies. Rev Saude Publica 2008; 42(6):992-998.

17. Victora CG, Huttly SR, Fuchs SC, Olinto MT. The role of conceptual frameworks in epidemiological analysis: a hierarchical approach. Int J Epidemiol 1997; 26(1):224-227.
18. Nogueira CMR. Conhecimento sobre aleitamento materno de parturientes e prática de aleitamento cruzado na Unidade Hospitalar e Maternidade Venâncio Raimundo de Souza - Horizonte - Ceará [dissertação]. Rio de Janeiro: Escola Nacional de Saúde Pública Sergio Arouca; 2008.

19. Boccolini CS, Carvalho ML, Oliveira MIC. Risk factors for cross nursing in two Brazilian cities. In: Anais do XVI Congreso Latinoamericano de Nutrición; 2012 nov 11-16; Havana, Cuba: Sociedad Latinoamericana de Nutrición; 2012.

20. Gartner LM, Morton J, Lawrence RA, Naylor AJ, O'Hare D, Schanler RJ, Eidelman AI; American Academy of Pediatrics Section on Breastfeeding. Breastfeeding and the use of human milk. Pediatrics 2005; 115(2):496506.

21. Sousa DA, Cerqueira-Santos E. Social networks and friendship relationships throughout the lifespan. Rev Psicopedagogia 2011; 28(85):53-66.

22. Stevenson W, Maton KI, Teti DM. Social support, relationship quality, and well-being among pregnant adolescents. J Adolesc 1999; 22(1):109-121.

23. Rondina RC, Gorayeb R, Botelho C. Psychological characteristics associated with tobacco smoking behavior. J Bras Pneumol. 2007; 33(5):592-601.

24. Secretaria Nacional Anti-drogas (SENAD). I Levantamento Nacional sobre os padrões de consumo de álcool na população brasileira. Brasília: SENAD; 2007.

25. Oliveira TR, Simões SMF. O consumo de bebida alcoólica pelas gestantes: um estudo exploratório. Esc Anna Nery Rev Enferm 2007; 11(4):632-638.

26. Broilo MC, Louzada ML, Drachler ML, Stenzel LM, Vitolo MR. Maternal perception and attitudes regarding healthcare professionals' guidelines on feeding practices in the child's first year of life. J Pediatr 2013; 89(5):485-491.

27. Heinig MJ, Follett JR, Ishii KD, Kavanagh-Prochaska $\mathrm{K}$, Cohen R, Panchula J. Barriers to compliance with infant-feeding recommendations among low-income women. J Hum Lact 2006; 22(1):27-38.

28. Olson BH, Horodynski MA, Brophy-Herb H, Iwanski KC. Health professionals' perspectives on the infant feeding practices of low income mothers. Matern Child Health J 2010; 14(1):75-85.

29. Vieira ACJ, Amaral DF, Pedrosa KJ, Ichisato SMT. Conhecimento das puérperas sobre aleitamento cruzado. Revista do Centro Universitário Barão de Mauá 2001; $1(2): 1-12$.

30. Sertório SC, Silva IA. The symbolic and utilitarian facets of pacifiers according to mothers. Rev Saude Publica 2005; 39(2):156-162.

31. Almeida JA, Novak FR. Breastfeeding: a nature-culture hybrid. J Pediatr 2004; 80(5 Supl.):S119-125.

Artigo apresentado em 01/09/2015

Aprovado em 17/11/2015

Versão final apresentada em 19/11/2015 